\title{
FOURIER COSINE TRANSFORMS WHOSE REAL PARTS ARE NON-NEGATIVE IN A STRIP
}

\author{
D. V. WIDDER ${ }^{1}$
}

1. Introduction. In a recent note [1] we obtained the Poisson integral representation of every function $u(x, y)$ which is positive and harmonic in a strip, $-1<y<1$. We here make use of this result to characterize those Fourier cosine transforms

$$
\int_{0}^{\infty} \cos (x+i y) t \phi(t) d t
$$

whose real parts are positive and integrable on $-\infty<x<\infty$ for each $y$ in $-1<y<1$. The characterizing condition on $\phi(t)$ is that $\phi(t) \cosh t$ (defined for $t<0$ so as to be even) should be real and positive definite. As an example, we have the classical equations

$$
\begin{array}{ll}
\frac{1}{z^{2}+1}=\int_{0}^{\infty} \cos z r e^{-r} d r, & z=x+i y, \\
u(x, y)=\operatorname{Re} \frac{1}{z^{2}+1}=\frac{x^{2}-y^{2}+1}{\left(x^{2}-y^{2}+1\right)^{2}+4 x^{2} y^{2}}>0, & |y|<1, \\
\int_{-\infty}^{\infty} u(x, y) d x=\pi, & |y|<1, \\
e^{-|r|} \cosh r=\frac{1}{2} \int_{-\infty}^{\infty} e^{i r t} d\left[U(t)+\frac{1}{\pi} \tan ^{-1} \frac{t}{2}\right] .
\end{array}
$$

Here $U(t)$ is zero for $t<0$ and unity for $t>0$, so that the integrator function in (2) is increasing and bounded. Thus $e^{-|r|} \cosh r$ is positive definite in confirmation of the theory. Equation (1) can be checked directly or will follow from Corollary 2 below.

An analogous result for the sine-transform is also obtained.

2. Positive integrable harmonic functions. In [1] the Poisson integral representation of functions positive and harmonic in a strip was obtained. If such functions are also integrable over the whole doublyinfinite lines of the strip they also have a simple Fourier integral representation, which we now obtain. We use $H$ and $L$ to denote the classes of harmonic and integrable (on the whole $x$-axis) functions,

Received by the editors October 6, 1964.

1 This research was supported by the Air Force Office of Scientific Research. 
respectively. Let us state Theorem 4 of [1], altered in notation only. Set

(3)

$$
Q(x, y)=\frac{1}{4} \frac{\cos \frac{\pi}{2} y}{\cosh \frac{\pi}{2} x+\sin \frac{\pi y}{2}}
$$

$$
=\frac{1}{\pi} \frac{d}{d x} \tan ^{-1} \quad\left(\frac{\sin \frac{\pi y}{2}+e^{\pi x / 2}}{\cos \frac{\pi y}{2}}\right) .
$$

Theorem A. A necessary and sufficient condition that $u(x, y)$ should be non-negative and harmonic in the strip $-1<y<1$ is that

$$
u(x, y)=\left[A e^{\pi x / 2}+B e^{-\pi x / 2}\right] \sin \frac{\pi y}{2}+\int_{-\infty}^{\infty} Q(x-t, y) d \alpha(t)
$$

$$
+\int_{-\infty}^{\infty} Q(x-t,-y) d \beta(t), \quad-1<y<1,
$$

where $A \geqq 0, B \geqq 0, \alpha(t)$ and $\beta(t)$ are nondecreasing.

To obtain our basic result we observe that $Q(x, y)$ is itself a positive definite function of $x$. This results from the fact that it is the Fourier transform of a positive function,

$$
Q(x, y)=\frac{1}{2 \pi} \int_{-\infty}^{\infty} e^{i x t} \frac{\sinh (1-y) t}{\sinh 2 t} d t, \quad-1<y<1 .
$$

See p. 36 of $[2]$.

THEOREM 1. A necessary and sufficient condition that $u(x, y) \in H$, $\in L, \geqq 0$ for $-1<y<1$ is that

(6) $u(x, y)=\int_{-\infty}^{\infty} e^{i x r} \frac{\sinh (1-y) r}{\sinh 2 r} g(r) d r+\int_{-\infty}^{\infty} e^{i x r} \frac{\sinh (1+y) r}{\sinh 2 r} h(r) d r$, where $g(r)$ and $h(r)$ are positive definite.

To prove this it will be sufficient to show that under the added condition

$$
\int_{-\infty}^{\infty} u(x, y) d x<\infty, \quad-1<y<1
$$


the representations (4) and (6) are identical. Since every term of (4) is nonnegative it is clear that the above inequality cannot hold unless $A=B=0$. From (3) we see that

$$
\int_{-\infty}^{\infty} Q(x, y) d x=\frac{1-y}{2}, \quad-1<y<1 .
$$

Hence by Fubini's theorem (7) can then hold if and only if the nondecreasing functions $\alpha$ and $\beta$ are also bounded. Then (7) takes the explicit form

$$
\int_{-\infty}^{\infty} u(x, y) d x=\frac{1-y}{2} \int_{-\infty}^{\infty} d \alpha(t)+\frac{1+y}{2} \int_{-\infty}^{\infty} d \beta(t)<\infty .
$$

Now substituting (5) in (4) we obtain

$$
\begin{aligned}
u(x, y)= & \frac{1}{2 \pi} \int_{-\infty}^{\infty} d \alpha(t) \int_{-\infty}^{\infty} e^{i(x-t) r} \frac{\sinh (1-y) r}{\sinh 2 r} d r \\
& +\frac{1}{2 \pi} \int_{-\infty}^{\infty} d \beta(t) \int_{-\infty}^{\infty} e^{i(x-t) r} \frac{\sinh (1+y) r}{\sinh 2 r} d r .
\end{aligned}
$$

In view of the boundedness of $\alpha$ and $\beta$ we may again apply Fubini's theorem to invert the order of integration, obtaining (6) with

$$
\begin{aligned}
& g(r)=\frac{1}{2 \pi} \int_{-\infty}^{\infty} e^{-i t r} d \alpha(t), \\
& h(r)=\frac{1}{2 \pi} \int_{-\infty}^{\infty} e^{-i t r} d \beta(t) .
\end{aligned}
$$

By Bochner's theorem [3] $g$ and $h$ are positive definite. This concludes the proof.

CoROLLARY 1. If to the conditions of the theorem is added that $u(x,-y)=u(x, y)$ they become necessary and sufficient that

$$
u(x, y)=\int_{-\infty}^{\infty} e^{i \pi r} \frac{\cosh y r}{\cosh r} g(r) d r, \quad-1<y<1,
$$

where $g(r)$ is positive definite.

For, from (6)

$$
u(x, y)=\frac{u(x, y)+u(x,-y)}{2}=\int_{-\infty}^{\infty} e^{i i r} \frac{\cosh y r}{\cosh r} \frac{g(r)+h(r)}{2} d r .
$$


The proof is concluded by an obvious change in notation.

A simple change of variable shows that if the strip $-1<y<1$ of Theorem 1 is replaced by $0<y<c$, then (6) becomes

(9) $u(x, y)=\int_{-\infty}^{\infty} e^{i x r} \frac{\sinh (c-y) r}{\sinh c r} g(r) d r+\int_{-\infty}^{\infty} e^{i x r} \frac{\sinh y r}{\sinh c r} h(r) d r$,

where $g$ and $h$ are positive definite.

3. The Fourier cosine transform. The precise statement of the result of $\$ 1$ follows.

TheOREM 2. The conditions

A. $u(x, y) \in H, \geqq 0, \in L(-\infty<x<\infty),-1<y<1$,

B. $u(-x, y)=u(x,-y)=u(x, y)$

are necessary and sufficient that

$$
u(x, y)=\operatorname{Re} \int_{0}^{\infty} \cos (x+i y) t \phi(t) d t, \quad-1<y<1,
$$

where $\phi(|t|) \cosh t$ is real and positive definite.

Under Conditions A and B, Corollary 1 shows that

$$
u(x, y)=\int_{-\infty}^{\infty} e^{i x r} \frac{\cosh y r}{\cosh r} g(r) d r, \quad-1<y<1,
$$

for some positive definite function $g(r)$. Denote the real and imaginary parts of $g$ by $g_{1}$ and $g_{2}$ respectively. Then $g_{1}$ is even and positive definite; $g_{2}$ is odd. By $\mathrm{B}, u(x, y)$ is even in $x$, so that

$$
\begin{aligned}
u(x, y) & =\int_{-\infty}^{\infty} \cos x r \frac{\cosh y r}{\cosh r} g_{1}(r) d r \\
& =\operatorname{Re} \int_{0}^{\infty} \cos (x+i y) r \phi(r) d r, \\
\phi(|r|) & =2 g_{1}(r) / \cosh r, \quad-\infty<r<\infty .
\end{aligned}
$$

This proves the sufficiency of Conditions A and B. The necessity follows easily.

Corollary 2. Under the Conditions A and B

$$
\int_{-\infty}^{\infty} u(x, y) d x=\pi \phi(0), \quad-1<y<1 .
$$

Write equation (10) as 


$$
u(x, y)=\int_{-\infty}^{\infty} e^{i \pi r} \frac{\sinh (1-y) r}{\sinh 2 r} g_{1}(r) d r+\int_{-\infty}^{\infty} e^{i x r} \frac{\sinh (1+y) r}{\sinh 2 r} g_{1}(r) d r .
$$

where

$$
g_{1}(r)=\frac{1}{2 \pi} \int_{-\infty}^{\infty} e^{-i t r} d \alpha(t) .
$$

Now apply equation (7) with $\alpha=\beta$ :

$$
\int_{-\infty}^{\infty} u(x, y) d x=\int_{-\infty}^{\infty} d \alpha(t)=2 \pi g_{1}(0)=\pi \phi(0) .
$$

In the example of $\S 1, \phi(0)=1$, so that equation (1) is established.

4. The Fourier sine-transform. A companion result to Theorem 2 is the following.

THEOREM 3. The conditions

A. $u(x, y) \in H, \in L(-\infty<x<\infty),-1<y<1$,

B. $u(x, y) \geqq 0,0<y<1$,

C. $u(-x, y)=-u(x,-y)=u(x, y)$

are necessary and sufficient that

(11) $u(x, y)=\operatorname{Im} \int_{0}^{\infty} \sin (x+i y) t \phi(t) d t, \quad-1<y<1$,

where $\phi(t) \sinh t$ is real positive definite ( $\phi$ being odd).

We first prove the necessity. Assume (11) with

$$
\phi(t) \sinh t=g(t) \int_{-\infty}^{\infty} e^{-i t r} d \alpha(r),
$$

where $\alpha(r)$ is nondecreasing and bounded. Then

$$
\begin{aligned}
u(x, y) & =\frac{1}{2} \int_{-\infty}^{\infty} \cos x t \frac{\sinh y t}{\sinh t} g(t) d t \\
& =\frac{\pi}{2} \int_{-\infty}^{\infty} \frac{\sin \pi y}{\cosh (x-r) \pi+\cos \pi y} d \alpha(r) .
\end{aligned}
$$

From (13) we may now verify Conditions A and B; from (12), Condition C.

Conversely, from (9) with $c=1$, we have for $0<y<1$

(14) $u(x, y)=\int_{-\infty}^{\infty} e^{i \pi r} \frac{\sinh (1-y) r}{\sinh r} g(r) d r+\int_{-\infty}^{\infty} e^{i x r} \frac{\sinh y r}{\sinh r} h(r) d r$, 
where $g$ and $h$ are positive definite. Assuming $u(x, y)$ odd in $y$, we see that $u(x, 0)=0$ (A and C). We now show that the first of the integrals (14) is identically zero. From (13) it is clear that it defines a non-negative harmonic function $v(x, y)$ in $0<y<2$. The second of the integrals (14) is harmonic in $-1<y<1$ and vanishes for $y=0$. From equation $(14), v(x, 0)=0$. But $v(x, 1)=0$. Hence we may apply the uniqueness result, Corollary 2.2 of [1], to conclude that

$$
v(x, y)=\left[A e^{\pi x}+B e^{-\pi x}\right] \sin \pi y, \quad A \geqq 0, B \geqq 0 .
$$

Since

$$
\int_{-\infty}^{\infty} v(x, y) d x \leqq \int_{-\infty}^{\infty} u(x, y) d x<\infty, \quad 0<y<1
$$

it follows by Condition A that $A=B=0$, so that $v$ vanishes identically.

Now writing $h=h_{1}+i h_{2}$ we have

$$
\begin{aligned}
u(x, y) & =\int_{-\infty}^{\infty} \cos x r \frac{\sinh y r}{\sinh r} h_{1}(r) d r \\
& =\operatorname{Im} \int_{0}^{\infty} \sin (x+i y) r \phi(r) d r, \\
\phi(r) & =-\phi(-r)=2 h_{1}(r) / \sinh r, \quad 0<r<\infty .
\end{aligned}
$$

This concludes the proof. As an example we may take

$$
\begin{aligned}
u(x, y) & =\frac{\pi}{2} \frac{\sin \pi y}{\cosh \pi x+\cos \pi y}=\frac{\pi}{2} \operatorname{Im} \tanh \pi(x+i y) \\
& =\operatorname{Im} \int_{0}^{\infty} \frac{\sin (x+i y) t}{\sinh t} d t .
\end{aligned}
$$

Here $\phi(t)=1 / \sinh t$, and the function 1 is real and positive definite.

5. Positive integrable harmonic functions in a half plane. In [4] we showed that $u(x, y)$ is harmonic, $\geqq 0$ and integrable in $x(-\infty<x$ $<\infty)$ for $0<y<\infty$ if and only if

$$
u(x, y)=\int_{-\infty}^{\infty} e^{i x r-y|r|} \psi(r) d r
$$

where $\psi(r)$ is positive definite. Under these conditions $u(x, y)$ satisfies the conditions of Theorem 1 in the strip $0<y<c$ for every $c>0$. Thus $u(x, y)$ has the two representations (9) and (15). It is perhaps 
useful to record the relations between the functions $g, h$, and $\psi$. By use of the identity

$$
\sigma^{-r|r|}=e^{-c|r|} \frac{\sinh y r}{\sinh c r}+\frac{\sinh (c-y) r}{\sinh c r}
$$

we see at once that

$$
g(r)=\psi(r), \quad h(r)=e^{-c|r|} \psi(r),
$$

so that $g$ is independent of $c$, and $h$ is an exponential multiple of $g$. As one would expect the first integral (9) tends to the integral (15) as $c \rightarrow+\infty$, the second approaches zero.

\section{REFERENCES}

1. D. V. Widder, Functions harmonic in a strip, Proc. Amer. Math. Soc. 12 (1961), 67-72.

2. F. Oberhettinger, Tabellen zur Fourier Transformation, Springer, Berlin, 1957.

3. S. Bochner, Lectures on Fourier integrals, Princeton Univ. Press, Princeton, N. J., 1959, pp. 92-96.

4. D. V. Widder, Functions of three variables which satisfy both the heat equation and Laplace's equation in two variables, J. Austral. Math. Soc. 3 (1963), 396-407.

HARVARD UNIVERSITY 